\title{
Biofilm and metallo beta-lactamase production among the strains of Pseudomonas aeruginosa and Acinetobacter spp. at a Tertiary Care Hospital in Kathmandu, Nepal
}

Bandana Baniya ${ }^{1}$, Narayan Dutt Pant ${ }^{2 *}$, Sanjeev Neupane ${ }^{3}$, Saroj Khatiwada ${ }^{4}$, Uday Narayan Yadav ${ }^{5}$, Nisha Bhandari ${ }^{6}$, Rama Khadka ${ }^{1}$, Sabita Bhatta ${ }^{7}$ and Raina Chaudhary ${ }^{7}$

\begin{abstract}
Introduction: Pseudomonas aeruginosa and Acinetobacter spp. are found to be associated with biofilm and metallo$\beta$-lactamase production and are the common causes of serious infections mainly in hospitalized patients. So, the main aims of this study were to determine the rates of biofilm production and metallo beta-lactamase production (MBL) among the strains of Pseudomonas aeruginosa and Acinetobacter spp. isolated from hospitalized patients.

Methods: A total of 85 P. aeruginosa isolates and 50 Acinetobacter spp. isolates isolated from different clinical specimens from patients admitted to Shree Birendra Hospital, Kathmandu, Nepal from July 2013 to May 2014 were included in this study. The bacterial isolates were identified with the help of biochemical tests. Modified Kirby-Bauer disc diffusion technique was used for antimicrobial susceptibility testing. Combined disc diffusion technique was used for the detection of MBL production, while Congo red agar method and tube adherence method were used for detection of biofilm production.

Results: Around $16.4 \%$ of P. aeruginosa isolates and $22 \%$ of the strains of Acinetobacter spp. were metallo $\beta$-lactamase producers. Out of 85 P. aeruginosa isolates, 23 (27.05\%) were biofilm producers according to tube adherence test while, only 13 (15.29\%) were biofilm producers as per Congo red agar method. Similarly, out of 50 Acinetobacter spp. 7 (14\%) isolates were biofilm producers on the basis of tube adherence test, while only 5 (10\%) were positive for biofilm production by Congo red agar method. Highest rates of susceptibility of $P$. aeruginosa as well as Acinetobacter spp. were seen toward colistin.
\end{abstract}

Conclusion: In our study, biofilm production and metallo beta-lactamase production were observed among Pseudomonas aeruginosa and Acinetobacter spp. However, no statistically significant association could be established between biofilm production and metallo beta-lactamase production.

Keywords: Biofilm, MBL, Pseudomonas aeruginosa, Acinetobacter spp.

\section{Introduction}

With the emergence of carbapenemase (mainly metallo$\beta$-lactamase) producing bacterial strains, the clinical utility of carbapenem as reserve drug is under threat [1].

\footnotetext{
*Correspondence: ndpant1987@gmail.com

2 Department of Microbiology, Grande International Hospital, Dhapasi, Kathmandu, Nepal

Full list of author information is available at the end of the article
}

Such bacterial strains have emerged all over the world and show high-level resistance to all $\beta$-lactams except aztreonam [2]. Antimicrobial resistance associated with biofilm production presents a serious threat in clinical practice in case of biofilm associated infections [3, 4]. Biofilm producers produce an extracellular matrix of polysaccharides which act as a protective jacket for the bacteria within biofilms, preventing diffusion of antibiotics, immune cells and host proteins [5]. Thus, it is 
of utmost importance to screen for biofilm production among clinical isolates. The first step in the treatment of biofilm associated infections is the detection of biofilms and it needs to be incorporated as a routine laboratory procedure. Antibiotics used for the treatment of such infections should be directed against the biofilms rather than the planktonic forms [6]. Pseudomonas aeruginosa and Acinetobacter spp. are the common causes of life threatening infections mainly in hospitalized patients [7]. In addition, biofilm and metallo- $\beta$-lactamase production among these bacteria may present as serious problem to the treatment of the infections caused by them. In this study, we determined the rates of biofilm production and MBL production among the strains of Pseudomonas aeruginosa and Acinetobacter spp. Further, we determined the antimicrobial susceptibility patterns of these organisms.

\section{Methods}

A total of 85 P. aeruginosa isolates and 50 Acinetobacter spp. isolates, isolated from different clinical specimens from patients admitted to Shree Birendra Hospital, Kathmandu, Nepal from July 2013 to May 2014 were included in this study. The bacterial isolates were identified on the basis of the microbiological procedures as described in the Bergey's manual of determinative bacteriology and were further evaluated for MBL production by combined disk diffusion method using imipenem and imipenem/ ethylenediaminetetraacetate discs. Similarly, biofilm production was detected by tube adherence method and Congo red agar method [8, 9]. Modified Kirby-Bauer disc diffusion technique was used for antimicrobial susceptibility testing following clinical and laboratory standards institute guidelines 2013. The bacteria showing resistance to at least three different classes of antibiotics were taken as MDR. Statistical package for the social sciences version 16.0. was used for data analysis. Chi square test was applied and $p<0.05$ was considered significant.

\section{Results}

Of the total $85 P$. aeruginosa isolates, 14 (16.4\%) were metallo $\beta$-lactamase producers, while of the 50 Acinetobacter spp. 11 (22\%) were metallo $\beta$-lactamase producers.

Out of 85 P. aeruginosa isolates, 23 (27.05\%) were biofilm producers according to tube adherence test, while only $13(15.29 \%)$ were biofilm producers as per Congo red agar method. Similarly, out of 50 Acinetobacter spp. 7 (14\%) isolates were biofilm producers on the basis of tube adherence test while, only $5(10 \%)$ were positive for biofilm production by Congo red agar method. Of the total 14 MBL producing $P$. aeruginosa isolates, only 2 were biofilm producers on the basis of Congo red agar method, whereas 4 were biofilm producers as per tube adherence method. Similarly, of total $11 \mathrm{MBL}$ producing strains of Acinetobacter spp., only 2 were biofilm producers on the basis of Congo red agar method, whereas 1 was biofilm producer as per tube adherence method. Highest rate of susceptibility of $P$. aeruginosa was seen toward colistin (83.5\%) followed by netilmycin (71.9\%). Lowest rate of susceptibility was seen toward cefepime (22.4\%). Highest rate of susceptibility of Acinetobacter spp. was seen toward colistin (74\%) followed by netilmicin (70\%). Lowest rate of susceptibility was seen toward ceftriaxone $(6 \%)$ (Table 1). Of the total P. aeruginosa isolates, 56 (65.8\%) were multidrug resistant, while of the total Acinetobacter spp. isolates, $45(90 \%)$ were multidrug resistant. Higher rates of drug resistance were seen among biofilm producers in comparison to biofilm non producers but the correlation was statistically insignificant.

Similarly, no statistically significant association between biofilm production and metallo beta-lactamase production could be established.

\section{Discussion}

Pseudomonas aeruginosa and Acinetobacter spp. are common causes of life threatening infections mainly in hospitalized patients. Further, the biofilm production and the metallo- $\beta$-lactamase production among these bacteria have further aggravated the problem. Infections with multidrug resistant $P$. aeruginosa and Acinetobacter spp. are of serious concern mainly in admitted patients [7].

In this study, $16.4 \%$ of $P$. aeruginosa were metallo $\beta$-lactamase producers which was in agreement with the finding by Kali et al. (16.3\%) [10]. Similar rate of MBL production among Acinetobacter spp. as in our study was also reported by Lee et al. (15.1\%) [11].

Table 1 Antibiotic susceptibility patterns of Acinetobacter spp. and $P$. aeruginosa

\begin{tabular}{lll}
\hline Antibiotics & \multicolumn{2}{l}{ Susceptible } \\
\cline { 2 - 3 } & Acinetobacter spp. (\%) & P. aeruginosa (\%) \\
\hline Piperacillin-tazobactam & 24 & 45.9 \\
Ceftazidime & 14 & 49.4 \\
Cefepime & 16 & 22.4 \\
Imipenem & 56 & 69.4 \\
Gentamicin & 22 & 48.2 \\
Amikacin & 22 & 50.6 \\
Netilmicin & 70 & 71.9 \\
Ciprofloxacin & 18 & 38.8 \\
Ofloxacin & 30 & 36.5 \\
Colistin & 74 & 83.5 \\
Cefotaxime & 14 & \\
Ceftriaxone & 6 & \\
Doxycycline & 24 & \\
\hline
\end{tabular}


Polymyxin B and colistin have been reported to demonstrate reasonable success in treatment of infections caused by MBL producing Acinetobacter spp. and $P$. aeruginosa. However, due to their high toxicity, polymyxins are used for the treatment of only serious infections caused by pan-resistant Gram negative bacilli [12]. A study in Thailand showed that all the multidrug resistant $P$. aeruginosa and Acinetobacter spp. were susceptible to polymyxin B and colistin [13].

Very high rates of biofilm production in comparison to our study were reported by Rewatkar and Wadher among the strains of $P$. aeruginosa $(90 \%$ by Congo red agar method and $83.33 \%$ by tube adherence method) [14] and by Badave and Kulkarni among the isolates of Acinetobacter spp. (65.2\%) [15].

In our study, no statistically significant association between biofilm production and metallo beta-lactamase production along with antimicrobial resistance could be established, which was in contrast to the findings by Heydari and Eftekhar [16] and Singhai et al. [17]. However, higher rates of drug resistance were seen among biofilm producers in comparison to biofilm non producers. Though higher numbers of biofilm producers were detected by tube adherence method in comparison to Congo red agar method but the correlation was statistically insignificant. The lack of significance might be due to small sample size taken in our study.

Though the higher numbers of biofilm producers were detected by tube adherence method in our study, due to the simplicity and cost effectiveness of the Congo red agar method [8] in comparison to tube adherence method, it is more appropriate for laboratory use in the developing countries like Nepal. In addition, its sensitivity and specificity have been reported to be $89 \%$ and $100 \%$ respectively, when compared to polymerase chain reaction as standard [9]. Similarly, those for tube adherence method were $100 \%$ [9].

\section{Limitations of the study}

Due to lack of resources we could not use molecular techniques in our study. Further, this is a uni-center study; multi-center study with large sample size would have generated more reliable results.

\section{Conclusions}

In our study, biofilm production and metallo beta-lactamase production were observed among Pseudomonas aeruginosa and Acinetobacter spp. However, no statistically significant association could be established between biofilm production and metallo beta-lactamase production.
Abbreviations

MBL: metallo beta-lactamase production; EDTA: ethylenediaminetetraacetic acid.

\section{Authors' contributions}

NDP designed the study, carried out the research works, analyzed data, and prepared the manuscript. BB, SN and UNY designed the study, carried out the research works, and analyzed the data. SK and NB contributed in analysis of the data. RK, SB, RC monitored the study. All authors read and approved the final manuscript.

\section{Author details \\ ${ }^{1}$ Department of Microbiology, Kantipur College of Medical Science, Sitapaila, Kathmandu, Nepal. ${ }^{2}$ Department of Microbiology, Grande International Hospital, Dhapasi, Kathmandu, Nepal. ${ }^{3}$ Central Department of Microbiology, Tribhuvan University, Kirtipur, Kathmandu, Nepal. ${ }^{4}$ Department of Biochemis- try, Modern Technical College, Lalitpur, Kathmandu, Nepal. ${ }^{5}$ Forum for Health Research and Development, Dharan, Nepal. ${ }^{6}$ Department of Biotechnology, University of Madras, Chennai, India. ${ }^{7}$ Department of Microbiology, Nepalese Army Institute of Health Sciences, Syanobharyang, Kathmandu, Nepal.}

\section{Acknowledgements}

The authors would like to thank, Kantipur college of medical science, Kathmandu, Nepal and Shree Birendra Hospital, Kathmandu, Nepal, for providing the opportunity to conduct this research. The authors would also like to thank all the patients and the technical staffs for their help during the study.

Competing interests

The authors declare that they have no competing interests.

Availability of data and materials

The data related to this study can be made available by the authors of this article if requested.

\section{Consent for publication}

Not applicable.

\section{Funding}

To conduct this study no fund was obtained from any sources.

\section{Publisher's Note}

Springer Nature remains neutral with regard to jurisdictional claims in published maps and institutional affiliations.

Received: 30 January 2017 Accepted: 30 October 2017

Published online: 02 November 2017

References

1. Mahajan G, Sheemar S, Chopra S, Kaur J, Chowdhary D, Makhija SK. Carbapenem resistance and phenotypic detection of carbapenemases in clinical isolates of Acinetobacter baumannii. Indian J Med Sci. 2011;65(1):18-25.

2. Andrade SS, Picao RC, Campana EH, Nicoletti AG, Pignatari AC, Gales AC. Influence of disk preparation on detection of metallo-beta-lactamase-producing isolates by the combined disk assay. J Clin Microbiol. 2007:45(6):2058-60

3. Hoiby N, Ciofu O, Johansen HK, Song ZJ, Moser C, Jensen PO, et al. The clinical impact of bacterial biofilms. Int J Oral Sci. 2011;3(2):55-65.

4. Hall MR, McGillicuddy E, Kaplan LJ. Biofilm: basic principles, pathophysiology, and implications for clinicians. Surg Infect. 2014;15(1):1-7.

5. Kokare CR, Chakraborty S, Khopade AN, Mahadik KR. Biofilm: importance and applications. IJBT. 2009;8:159-68.

6. Kostakioti M, Hadjifrangiskou M, Hultgren SJ. Bacterial biofilms: development, dispersal, and therapeutic strategies in the dawn of the postantibiotic era. Cold Spring Harb Perspect Med. 2013;3(4):a010306. 
7. Navon-Venezia S, Ben-Ami R, Carmeli Y. Update on Pseudomonas aeruginosa and Acinetobacter baumannii infections in the healthcare setting. Curr Opin Infect Dis. 2005;18(4):306-13.

8. Freeman DJ, Falkiner FR, Keane CT. New method for detecting slime production by coagulase negative staphylococci. J Clin Pathol. 1989;42(8):872-4.

9. Oliveira A, Cunha Mde L. Comparison of methods for the detection of biofilm production in coagulase-negative staphylococci. BMC Res Notes. 2010;3(260):1756-60.

10. Kali A, Srirangaraj S, Kumar S, Divya HA, Kalyani A, Umadevi S. Detection of metallo-beta-lactamase producing Pseudomonas aeruginosa in intensive care units. Australas Med J. 2013;6(12):686-93.

11. Lee K, Ha GY, Shin BM, Kim JJ, Kang JO, Jang SJ, et al. Metallo-beta-lactamase-producing Gram-negative bacilli in Korean Nationwide Surveillance of Antimicrobial Resistance group hospitals in 2003: continued prevalence of VIM-producing Pseudomonas spp. and increase of IMP-producing Acinetobacter spp. Diagn Microbiol Infect Dis. 2004;50(1):51-8.
12. Lee SY, Kuti JL, Nicolau DP. Polymyxins: older antibiotics for a new threat. Conn Med. 2006;70(1):25-8.

13. Koomanachai P, Tiengrim S, Kiratisin P, Thamlikitkul V. Efficacy and safety of colistin (colistimethate sodium) for therapy of infections caused by multidrug-resistant Pseudomonas aeruginosa and Acinetobacter baumannii in Siriraj Hospital, Bangkok, Thailand. Int J Infect Dis. 2007;11(5):402-6.

14. Rewatkar AR, Wadher BJ. Staphylococcus aureus and Pseudomonas aeruginosa-biofilm formation methods. IOSR-JPBS. 2013;8(5):36-40.

15. Badave GK, Kulkarni D. Biofilm producing multidrug resistant Acinetobacter baumannii: an emerging challenge. J Clin Diagn Res. 2015;9(1):1.

16. Heydari S, Eftekhar F. Biofilm formation and B-lactamase production in burn isolates of Pseudomonas aeruginosa. Jundishapur J Microbiol. 2015:8(3):e15514.

17. Singhai M, Rawat V, Goyal R. Concomitant detection of biofilm and metallo-beta-lactamases production in Gram-negative bacilli. Indian Pathol Microbiol. 2013;56(3):276-8.

\section{Submit your next manuscript to BioMed Central and we will help you at every step:}

- We accept pre-submission inquiries

- Our selector tool helps you to find the most relevant journal

- We provide round the clock customer support

- Convenient online submission

- Thorough peer review

- Inclusion in PubMed and all major indexing services

- Maximum visibility for your research

Submit your manuscript at www.biomedcentral.com/submit
() Biomed Central 